\title{
Inovação e gestão pública: uma análise da produção científica internacional
}

\section{Innovation and public management: an analysis of international scientific production}

\author{
SÉRGIO HENRIQUE DE OLIVEIRA LIMA, \\ Universidade Federal do Ceará e Universidade Federal do Cariri \\ LUÍS FELIPE CÂNDIDO \\ Universidade Federal do Ceará \\ IVELTYMA IBIAPINA \\ Universidade Federal do Ceará \\ ÁURIO LÚCIO LEOCÁDIO \\ Universidade Federal do Ceará
}

\section{RESUMO}

Diversos desafios têm levado as organizações na esfera da administração pública a inovar na prestação de seus serviços e na gestão de seus processos. Neste contexto, o objetivo do presente trabalho foi realizar um mapeamento da produção científica internacional sobre inovação e gestão pública por meio de um estudo bibliométrico. Para atingir tal objetivo, foi levantada e uma base de análise de 673 artigos. Os resultados mostraram a consolidação da produção científica no campo nos últimos anos, além de confirmar os pressupostos das leis bibliométricas de Lotke e Bradford, ao se observar uma concentração desproporcional desta produção em alguns autores e periódicos, respectivamente. Ademais, a visualização e a análise de redes bibliométricas permitiram identificar as principais temáticas de pesquisas no campo. Algumas limitações e sugestões de pesquisas futuras são discutidas para o desenvolvimento dos estudos no campo.

Palavras-chave: Administração pública. Bibliometria. Sociometria. Coautoria. VOSviewer.

\section{AbSTRACT}

Several challenges have led organizations in the public administration sphere to innovate in the provision of their services and in the management of their processes. In this context, the 
objective of the present paper was to map the international scientific production on innovation and public management through a bibliometric study. To achieve this goal, was raised and an analysis base of 673 articles. The results showed the consolidation of the scientific production in the field in recent years, in addition to confirming the assumptions of bibliometric laws Lotke and Bradford, to observe a disproportionate concentration of production in some authors and journals, respectively. Furthermore, visualization and analysis of bibliometric networks have identified the main topics of research in the field. Some limitations and suggestions for future research are discussed for the development of field studies.

Keywords: Public administration. Bibliometric. Sociometry. Co-author.

\section{INTRODUÇÃo}

Organizações públicas e seus atores têm enfrentado desafios de legitimidade crescentes, como questões voltadas à sustentabilidade, mobilidade urbana, desemprego, envelhecimento da população, revitalização de áreas urbanas, entre outros (DE VRIES, TUMMERS; BEKKERS, 2018).

Tais desafios, frequentemente ampliados por restrições orçamentárias, têm compelido a Administração Pública a inovar, ou seja, desenvolver e difundir novas práticas no sentido de atender aos interesses da população (OSBORNE; BROWN, 2011). Além de atender a esses interesses, inovação fortalece o desenvolvimento econômico, social e intelectual (PEREIRA; VIEIRA; DAMIÃO, 2018) das entidades públicas.

Neste sentido, o objetivo do presente estudo foi realizar um mapeamento da produção científica internacional sobre inovação e gestão pública. Por meio da análise da produção de autores e suas citações, periódicos, países e áreas temáticas, bem como das redes de colaboração que eles estabelecem espera-se exceder o escopo de estudos bibliométricos tradicionalmente empreendidos, razão por que se atribui relevância ao presente artigo.

Alguns estudos já realizaram esforços relacionados, como revisões sistemáticas da literatura internacional sobre inovação na gestão pública (LIMA; VARGAS, 2012; BRANDÃO; BRUNO-FARIA, 2013), 
mas com abordagens diferentes das que foram aqui realizadas, no que o presente estudo complementa a literatura atual sobre o tema.

\section{REVISÃo DE LITERATURA}

Nesta seção são abordados e discutidos os conceitos relacionados à inovação e gestão pública, além dos estudos bibliométricos e seus indicadores.

\subsection{Inovação e gestão pública}

Debates em torno da inovação na Administração Pública se intensificaram com o surgimento da "Nova Administração Pública" - há aproximadamente quarenta anos - uma vez que a agenda proposta, de redução do crescimento e do tamanho do governo, da desburocratização e de privatizações, precisaria coexistir com avanços e inovações em tecnologia da informação (HOOD, 1991).

Embora os princípios, definições e critérios de mensuração da inovação no setor privado possam ser, em certa medida, aplicados à administração pública (ARUNDEL; BLOCH; FERGUSON, 2019), o desenvolvimento e a difusão de inovações neste campo demandam processos, estruturas e iniciativas bastante diferentes (DE VRIES, TUMMERS; BEKKERS, 2018), talvez pela miríade de stakeholders envolvidos, como os próprios cidadãos, as agências estatais em diversos níveis governamentais, e corporações privadas que são influenciadas pelas iniciativas públicas de inovação (AXELSON; NETZ; SANDSTRÖM, 2017; ROCHA; SANTOS; VIEIRA, 2018).

Diversos estudos têm investigado o fenômeno da inovação no setor público, focando em seus principais serviços, como educação (SERDYUKOV, 2017), saúde (BOSSERT, 1998), mobilidade urbana (SMITH; SOCHOR; KARLSSON, 2019) e segurança pública (RUEDIGER, 2013). Outros procuram analisar as barreiras à inovação no setor público (BARTON CUNNINGHAM; KEMPLING, 2009; DEMIRCIOLGU, 2018; FASSAUER; SCHIRMER, 2008), ou no gerenciamento dos processos pelos quais a inovação penetra a estrutura da burocracia pública (AGGER; SØRENSEN, 2018), na busca de se equilibrar os princípios de eficiência, transparência e justiça com as necessidades de bem-estar e equidade (VOLACU, 2017). 
Assim, com o desenvolvimento deste campo de conhecimento, verifica-se oportuno mapear as características de sua produção científica, empreendendo uma abordagem bibliométrica.

\subsection{Análise bibliométrica e seus indicadores}

Segundo Bufrem e Prates (2005), a bibliometria trata da mensuração e evidenciação de informações acerca do conhecimento científico publicado. Procura analisar estatisticamente características de publicações escritas e descrever e prognosticar a divulgação científica em determinado campo, identificando comportamentos explicados pelas leis de Lotka, de Bradford e de Zipf (FERREIRA et al., 2019; MACHADO JÚNIOR et al., 2016). Pela proposição original da Lei de Lotka, o número de autores que subscrevem quantidade " $\mathrm{n}$ " de publicações em uma determinada área é de aproximadamente " $1 / \mathrm{n}^{2 \text { " }}$ daqueles que publicam somente um artigo (ALVARADO, 2008). No que diz respeito a periódicos, a lei de Bradford "permite, mediante a medição da produtividade das revistas, estabelecer o núcleo e as áreas de dispersão sobre um determinado assunto (...)" (VANTI, 2002, p. 153). Ao se dividir a produção acumulada de um campo científico em grupos de journals (zonas) com a mesma quantidade de documentos publicados, as primeiras zonas concentrarão número menor de periódicos, e as zonas seguintes, sucessivamente mais periódicos. Já a lei de Zipf enuncia que as palavras com maior ocorrência em um corpo de texto são indicativas de sua temática central (COSTA SANTOS, 2009).

Com o desenvolvimento no campo das ciências da informação, análises mais complexas têm surgido a complementar estas leis originais, viabilizando o mapeamento de determinado campo científico através de análises sociométricas, como redes de coautoria, de co-citação, e de acoplamento bibliográfico (BUFREM; PRATES, 2005). Redes de coautoria mostram como pesquisadores estão conectados em função da quantidade de publicações que produzem em conjunto (VAN ECK; WALTMAN, 2014). Duas obras são ditas co-citadas quando uma terceira as cita simultaneamente (SMALL, 1973); ou seja, quanto maior o número de documentos em que duas publicações são citadas em conjunto, mais forte é a relação de co-citação entre elas. Duas obras são bibliograficamente acopladas quando uma terceira é citada simultaneamente por ambas (KESSLER, 1963); assim, quanto mais referências dois papers compartilham, maior o seu acoplamento bibliográfico (VAN ECK; WALTMAN, 2014). Redes 
de co-ocorrência de palavras-chave apresentam a frequência com que duas palavras-chave são simultaneamente utilizadas, sugerindo temáticas de pesquisa.

\section{Procedimentos metodológicos}

Para o alcance do objetivo do estudo, adotou-se a abordagem da bibliometria. Para tanto, foram definidos critérios para consulta à base de dados escolhida (Scopus) e definição da amostra a ser analisada, conforme apresentado na figura 1 .

\section{Figura 1 - Processo de busca sistemática}

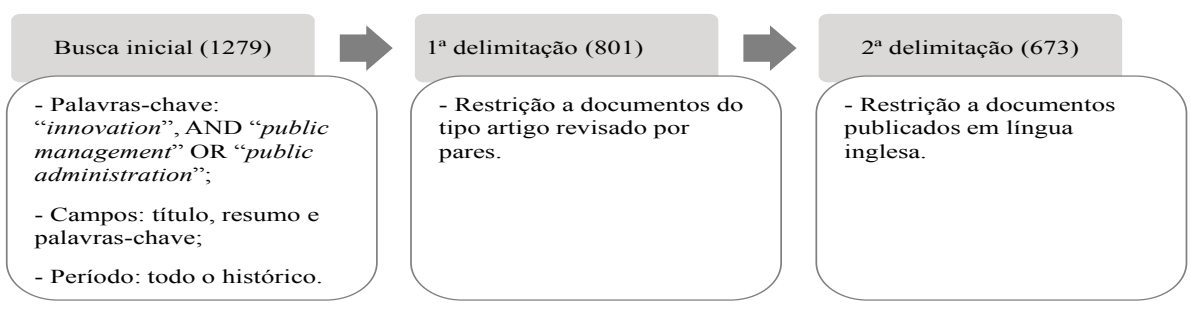

Fonte: elaborado pelos autores.

A consulta foi realizada em fevereiro de 2019. Como termos-chave de busca, adotaram-se as expressões "innovation", "public management" e "public administration", seguindo os operadores booleanos descritos na figura 1.

A expressão "public policy" não foi empregada na busca porque sua utilização elevaria em muito o tamanho da amostra, impedindo a adequada visualização das redes discutidas, e relevante parcela deste acréscimo são estudos sobre inovação fora da administração pública que mencionam, em seus campos de metadados, implicações para "políticas públicas".

Restringiu-se a amostra a documentos do tipo artigo revisado por pares, publicados em língua inglesa, gerando uma base de análise de 673 artigos. Considera-se que esta é uma base de dados suficientemente representativa para esboçar um útil mapa da pesquisa no campo.

Dois esforços foram empreendidos no estudo: (i) o mapeamento das características bibliográficas descritivas da amostra, a exemplo da evolução quantitativa da pesquisa no campo, dos autores, obras e periódicos de maior destaque; (ii) geração e análise de redes biblio- 
métricas, o que foi realizado com o suporte do software VOSviewer ${ }^{\odot}$ (versão 1.6.10).

Em bibliometria, algoritmos de visualização de redes empregam uma entre três abordagens mais comuns: distance-based, graph-based e timeline-based. O VOSviewer ${ }^{\odot}$ utiliza a primeira destas, ou seja, a força da relação entre os elementos de uma rede é indicada pela distância entre eles, de modo que quanto menor esta distância, mais forte é a sua relação, ou seja, a sua similaridade (VAN ECK; WALTMAN, 2014).

\section{RESUlTAdos E DiscusSões}

Nesta seção discutem-se primeiramente informações bibliográficas descritivas obtidas da amostra. Em seguida, são realizadas análises de redes bibliométricas de coautoria, de co-citação, de acoplamento bibliográfico e de co-ocorrência de palavras-chave.

\subsection{Evolução da produção no campo}

Os 673 documentos que constituíram a amostra do estudo foram subscritos por 1274 diferentes autores ou coautores e publicados em 334 periódicos. A evolução da quantidade de artigos publicados na base Scopus está apresentada na figura 2.

Figura 2 - Evolução da produção científica internacional sobre inovação e gestão pública

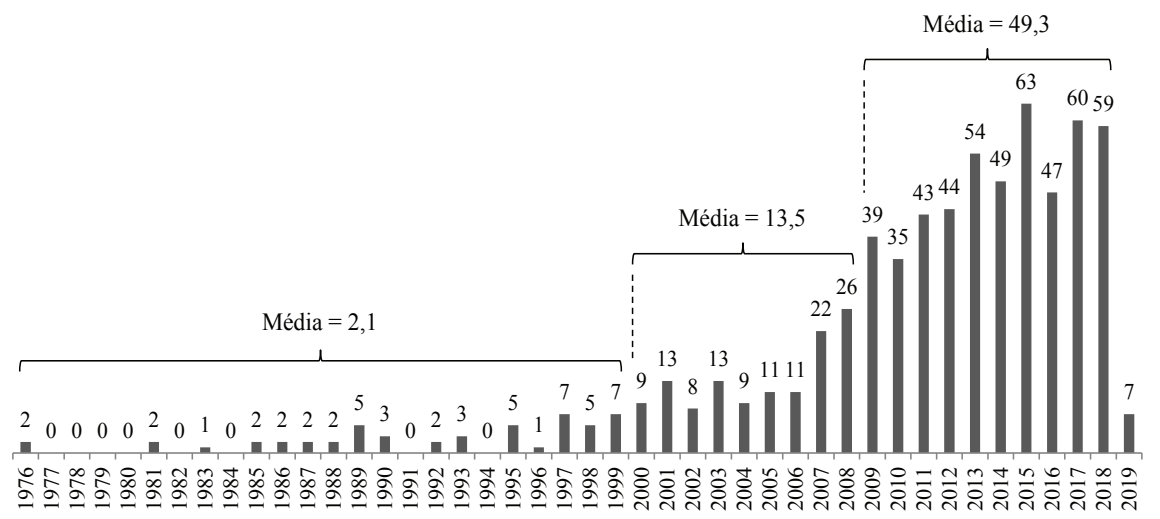

Fonte: elaborado pelos autores (dados da pesquisa).

O período completo da amostra (1976 a 2019) pode ser subdividido em três fases: (i) até 1999, em que houve uma média anual 
de 2,1 artigos publicados; (ii) entre 2000 e 2008, em que tal média se elevou para 13,5 publicações; e (iii) a partir de 2009, quando houve um salto para mais de 49 papers publicados anualmente. Observa-se ainda a estabilização da tendência de crescimento no volume de pesquisas, denotando o amadurecimento do campo.

Os primeiros artigos da amostra foram publicados em 1976, ambos na primeira edição do periódico Canadian Public Administration (v. 19, n.1). Embora pioneiros na base de dados aqui analisada, tais trabalhos não podem ser considerados seminais: juntos, receberam apenas três citações na Scopus no período analisado. Por outro lado, isto não lhes suprime o mérito de tratar mudanças administrativas na estrutura organizacional governamental - o primeiro em âmbito federal, e o segundo, provincial - como inovações na administração pública.

\subsection{Principais periódicos}

Para a avaliação dos periódicos que mais publicaram no período, segmentou-se a base de dados em três zonas ou faixas, cada uma delas contendo aproximadamente um terço de todos os artigos publicados no período de análise, conforme apresentado na tabela 1 .

\section{Tabela 1 - Principais periódicos}

\begin{tabular}{|c|c|c|c|c|c|c|c|c|}
\hline Periódico & $\begin{array}{c}\text { Até } \\
201 \\
4\end{array}$ & $\begin{array}{c}201 \\
5\end{array}$ & $\begin{array}{c}201 \\
6\end{array}$ & $\begin{array}{c}201 \\
7\end{array}$ & $\begin{array}{c}201 \\
8\end{array}$ & $\begin{array}{c}201 \\
9\end{array}$ & $\begin{array}{c}\text { Tota } \\
1 \\
\text { Gera } \\
1 \\
\end{array}$ & $\begin{array}{c}\% \\
\text { Artigos }\end{array}$ \\
\hline Public Administration Review & 22 & 2 & 2 & 4 & 1 & & 31 & \multirow{16}{*}{$\begin{array}{c}32,7 \% \\
\left(\begin{array}{c}\text { Zona } \\
1)\end{array}\right.\end{array}$} \\
\hline Canadian Public Administration & 17 & 1 & & 3 & 1 & & 22 & \\
\hline Australian Journal of Public Administration & 12 & 3 & 2 & 1 & 4 & & 22 & \\
\hline Public Management Review & 14 & 1 & & 2 & 1 & & 18 & \\
\hline International Journal of Public Administration & 10 & 2 & 1 & & 1 & & 14 & \\
\hline International Review of Administrative Sciences & 7 & & 1 & 1 & 3 & 1 & 13 & \\
\hline Public Administration & 9 & 1 & 1 & & 2 & & 13 & \\
\hline International Review of Public Administration & 10 & 1 & & 2 & & & 13 & \\
\hline Innovation Journal & 7 & 4 & & & & & 11 & \\
\hline $\begin{array}{l}\text { Journal of Public Administration Research and } \\
\text { Theory }\end{array}$ & 8 & 2 & 1 & & & & 11 & \\
\hline American Review of Public Administration & 8 & & 1 & 1 & & & 10 & \\
\hline $\begin{array}{l}\text { International Journal of Public Sector } \\
\text { Management }\end{array}$ & 9 & & & & & & 9 & \\
\hline Information Polity & 6 & 2 & & & 1 & & 9 & \\
\hline Public Administration and Development & 6 & 2 & & & & & 8 & \\
\hline Governance & 8 & & & & & & 8 & \\
\hline International Public Management Journal & 6 & 1 & & & 1 & & 8 & \\
\hline $\begin{array}{l}\text { Revistas com } 2 \text { a } 7 \text { papers publicados no período } \\
\text { (72) }\end{array}$ & 121 & 23 & 22 & 23 & 15 & 3 & 207 & $30,8 \%$ \\
\hline Revistas com 1 paper publicado no período (246) & 157 & 18 & 16 & 23 & 29 & 3 & 246 & $36,6 \%$ \\
\hline Total & 437 & 63 & 47 & 60 & 59 & 7 & 673 & $100,0 \%$ \\
\hline
\end{tabular}

Fonte: elaborado pelos autores (dados da pesquisa). 
São detalhados os dezesseis journals pertencentes à primeira zona. Cada um deles publicou pelo menos oito artigos, totalizando 220 documentos, ou 32,7\% da amostra do estudo (com média de quase catorze por journal). Cabe maior destaque para o Public Administration Review, o Canadian Public Administration, o Australian Journal of Public Administration e o Public Management Review. A segunda zona é formada por outros 72 periódicos que publicaram entre dois e sete documentos, o que corresponde a aproximadamente $31 \%$ de toda a amostra (e média de menos de três artigos por revista). Journals com apenas um documento publicado no período constituem cerca de $36 \%$ da base de dados e foram considerados pertencentes à terceira zona de dispersão (246 periódicos).

Da dispersão observada acima, verificam-se atendidos os pressupostos da lei de Bradford, uma vez que se verificou grande concentração de artigos em poucos periódicos na chamada primeira zona, e um aumento da dispersão nas zonas seguintes.

\subsection{Obras de maior impacto}

A tabela 2 apresenta as obras mais citadas dentre aquelas que constituem a amostra. Assim como para periódicos, aqui também se analisou a dispersão em três zonas, entretanto, tomando-se a quantidade de citações que cada uma das 673 obras obteve.

As obras do primeiro grupo acima representam pouco mais de $3 \%$ do total de artigos analisados e juntas receberam um terço das 8523 citações da amostra, constituindo a primeira zona de artigos de maior impacto. A segunda zona é formada por 75 artigos, enquanto a terceira, por 576. Estes números evidenciam a dispersão das citações, evidenciando um pequeno grupo de autores cujas obras são as de maior impacto neste campo emergente.

\subsection{Principais autores}

A tabela 3 mostra a afiliação de cada autor com três ou mais artigos na base. 
Tabela 2 - Obras de maior impacto

\begin{tabular}{|c|c|c|c|c|}
\hline Autor(es) (Ano) & Título do artigo & Periódico & $\begin{array}{c}\text { Qtde } \\
\text { Citações }\end{array}$ & $\begin{array}{c}\% \\
\text { Acum } \\
\end{array}$ \\
\hline Hicks D. (2012) & $\begin{array}{l}\text { Performance-based university } \\
\text { research... }\end{array}$ & Research Policy & 278 & $3,3 \%$ \\
\hline Bossert T. (1998) & $\begin{array}{l}\text { Analyzing the decentralization of } \\
\text { health... }\end{array}$ & $\begin{array}{l}\text { Social Science and } \\
\text { Medicine }\end{array}$ & 218 & $5,8 \%$ \\
\hline $\begin{array}{l}\text { Del Brio J.A., } \\
\text { Junquera B. (2003) }\end{array}$ & $\begin{array}{l}\text { A review of the literature on } \\
\text { environmental... }\end{array}$ & Technovation & 187 & $8,0 \%$ \\
\hline $\begin{array}{l}\text { Tolbert C.J., } \\
\text { Mossberger K., } \\
\text { McNeal R. (2008) }\end{array}$ & $\begin{array}{l}\text { Institutions, policy innovation, and } \\
\text { e-government in... }\end{array}$ & $\begin{array}{l}\text { Public Administration } \\
\text { Review }\end{array}$ & 169 & $10,0 \%$ \\
\hline Borins S. (2000) & $\begin{array}{l}\text { Loose cannons and rule breakers, or } \\
\text { enterprising leaders? ... }\end{array}$ & $\begin{array}{l}\text { Public Administration } \\
\text { Review }\end{array}$ & 159 & $11,9 \%$ \\
\hline $\begin{array}{l}\text { Walker R.M., } \\
\text { Damanpour F., } \\
\text { Devece C.A. (2011) }\end{array}$ & $\begin{array}{l}\text { Management innovation and } \\
\text { organizational performance... }\end{array}$ & $\begin{array}{l}\text { Journal of Public } \\
\text { Administration } \\
\text { Research and Theory }\end{array}$ & 149 & $13,6 \%$ \\
\hline $\begin{array}{l}\text { Arts B., Tatenhove } \\
\text { J.V. (2004) }\end{array}$ & $\begin{array}{l}\text { Policy and power: A conceptual } \\
\text { framework between... }\end{array}$ & Policy Sciences & 149 & $15,4 \%$ \\
\hline $\begin{array}{l}\text { Cajaiba-Santana G. } \\
(2014)\end{array}$ & $\begin{array}{l}\text { Social innovation: Moving the field } \\
\text { forward.... }\end{array}$ & $\begin{array}{l}\text { Technological } \\
\text { Forecasting and Social } \\
\text { Change }\end{array}$ & 144 & $17,0 \%$ \\
\hline Walker R.M. (2006) & $\begin{array}{l}\text { Innovation type and diffusion: An } \\
\text { empirical... }\end{array}$ & Public Administration & 124 & $18,5 \%$ \\
\hline $\begin{array}{l}\text { Farazmand A. } \\
\text { (1999) }\end{array}$ & $\begin{array}{l}\text { Globalization and public } \\
\text { administration }\end{array}$ & $\begin{array}{l}\text { Public Administration } \\
\text { Review }\end{array}$ & 124 & $20,0 \%$ \\
\hline \multicolumn{3}{|c|}{ Outras obras com 75 citações ou mais (12) } & 1155 & $33,5 \%$ \\
\hline $2^{\circ}$ grupo & \multicolumn{2}{|c|}{75 artigos - entre 23 e 74 citações } & 2867 & $67,1 \%$ \\
\hline \multirow[t]{2}{*}{$3^{\circ}$ grupo } & \multicolumn{2}{|c|}{576 artigos - até 22 citações (145 não citados) } & 2800 & $100,0 \%$ \\
\hline & & Total & 8523 & \\
\hline
\end{tabular}

Fonte: elaborado pelos autores (dados da pesquisa).

Sandford Borins, da University of Toronto, Canadá, é o autor com maior número de artigos (oito), embora o último deles tenha sido publicado há cinco anos. Antonello Zanfei (University of Urbino, Itália) e Richard M. Walker (University of Hong Kong) também merecem destaque com seis publicações. Segundo este critério, Itália e Dinamarca aparecem como os países mais representativos, com três autores prolíficos, cada.

De modo geral, estes dezenove autores (ou 1,5\% dos 1274 presentes na base) publicaram 75 trabalhos (ou 11\% do total de 673 em análise). Por outro lado, 1189 autores, o equivalente a 93,3\%, subscreveram apenas um dos 673 artigos analisados. Estes dados confirmam os pressupostos da lei de Lotka, uma vez que apenas uma pequena "elite" de autores publicou mais de três papers no campo. 
Tabela 3 - Principais autores, instituições e países

(critério quantitativo, quantidade de artigos publicados)

\begin{tabular}{|c|c|c|c|}
\hline Autores & $\begin{array}{l}\text { Qtde } \\
\text { Artigos }\end{array}$ & Afiliação mais recente & País \\
\hline Borins S. & 8 & University of Toronto & Canadá \\
\hline Zanfei A. & 6 & University of Urbino & Itália \\
\hline Walker R.M. & 6 & University of Hong Kong & Hong Kong \\
\hline Torfing J. & 5 & Roskilde University & Dinamarca \\
\hline Sorensen E. & 4 & Roskilde University & Dinamarca \\
\hline Palm K. & 4 & Mid Sweden University & Suécia \\
\hline Hansen M.B. & 4 & Aalborg University & Dinamarca \\
\hline Hickey G.M. & 4 & McGill University & Canadá \\
\hline Arduini D. & 4 & University of Urbino & Itália \\
\hline Vigoda-Gadot E. & 3 & University of Haifa & Israel \\
\hline Shoham A. & 3 & University of Haifa & Israel \\
\hline Yang K. & 3 & $\begin{array}{l}\text { Renmin University of China \& } \\
\text { Florida State University }\end{array}$ & China \& EUA \\
\hline Mele V. & 3 & Bocconi University & Itália \\
\hline Lilja J. & 3 & Mid Sweden University & Suécia \\
\hline Karo E. & 3 & Tallinn University of Technology & Estônia \\
\hline Kattel R. & 3 & $\begin{array}{l}\text { Tallinn University of Technology \& } \\
\text { University College London }\end{array}$ & $\begin{array}{l}\text { Estônia \& } \\
\text { Inglaterra }\end{array}$ \\
\hline Ferlie E. & 3 & King's Business School & Inglaterra \\
\hline Kinder T. & 3 & $\begin{array}{l}\text { Eddleston Innovation Ltd \& } \\
\text { University of Edinburgh (até 2016) }\end{array}$ & Escócia \\
\hline Fernandez S. & 3 & $\begin{array}{l}\text { Indiana University \& University of } \\
\text { Johannesburg }\end{array}$ & $\begin{array}{l}\text { EUA \& África } \\
\text { do Sul }\end{array}$ \\
\hline
\end{tabular}

Fonte: elaborado pelos autores (dados da pesquisa).

\subsection{Redes de coautoria}

A figura 4 apresenta as principais redes de coautoria dos 673 artigos da base de dados. Foram considerados os 104 autores com pelo menos quarenta citações no período analisado.

O tamanho de cada círculo (nó) é proporcional ao número de citações do autor correspondente na base. Identificaram-se 50 clusters de colaboração e 84 links de relacionamento entre autores. Destacam-se na figura os doze agrupamentos formados por três ou mais autores. Os grupos liderados 
Figura 4 - Redes de coautoria

clifton $n$.

$$
\text { brown I. cooper j. }
$$

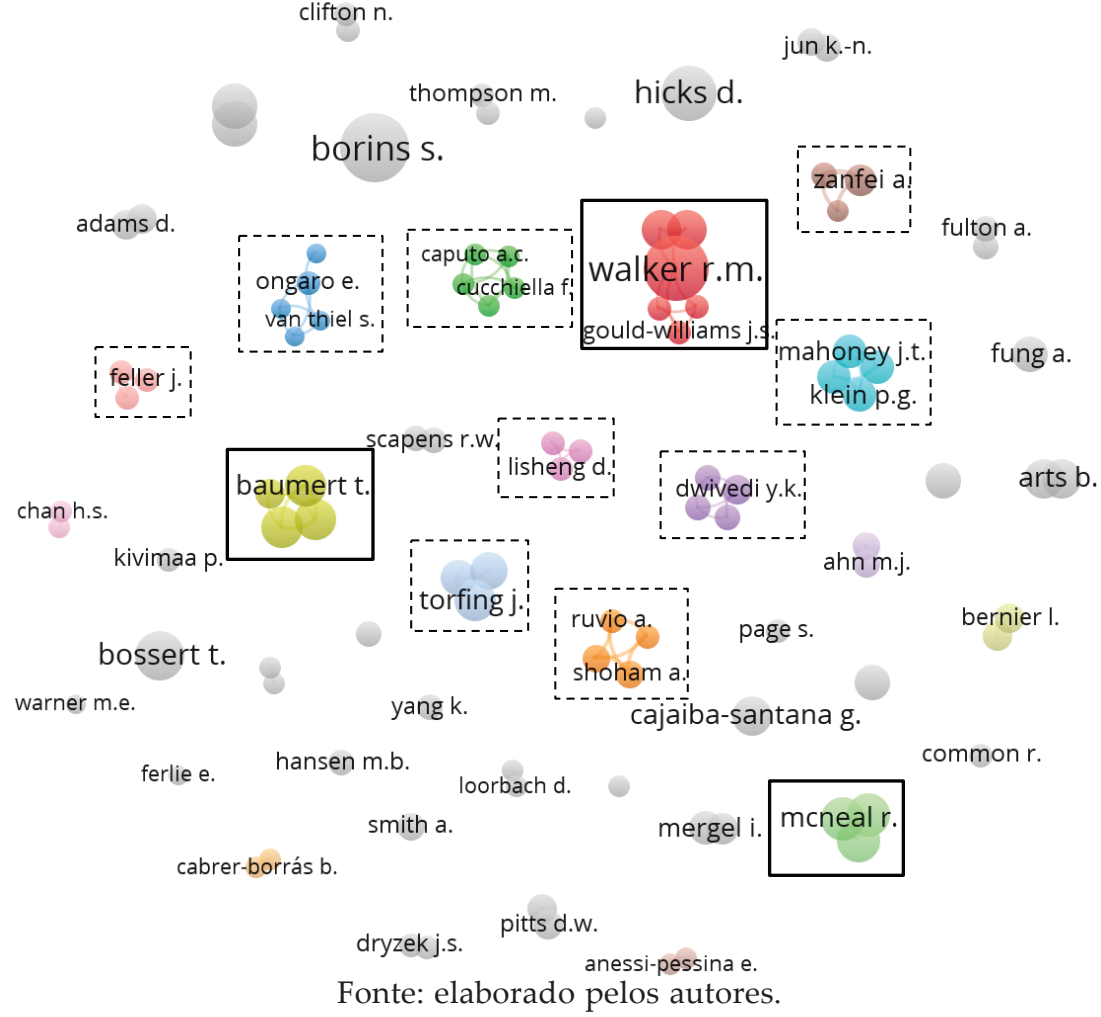

por Richard M. Walker, Thomas Baumert e Ramona McNeal são os grupos de maior impacto em termos de citações. Veem-se ainda alguns dos dezenove clusters formados por autor único, e cujas obras tiveram grande impacto. São os casos de Sandford Borins, Diana Hicks e Thomas Bossert.

\subsection{Rede de co-citação}

A figura 5 apresenta as relações de co-citação entre referências citadas pelos artigos analisados. De modo direto, esta rede apresenta as principais fontes a que os autores da base de dados recorreram para fundamentar seus estudos. São exibidas as obras com pelo menos cinco citações, o que gerou uma rede de co-citação com 26 nós 
distribuídos em quatro grupos. O tamanho do nó indica o número de citações que a respectiva obra de referência recebeu. Assim, obras agrupadas em um mesmo cluster têm mais forte relação de co-citação.

Figura 5 - Redes de co-citação de publicações

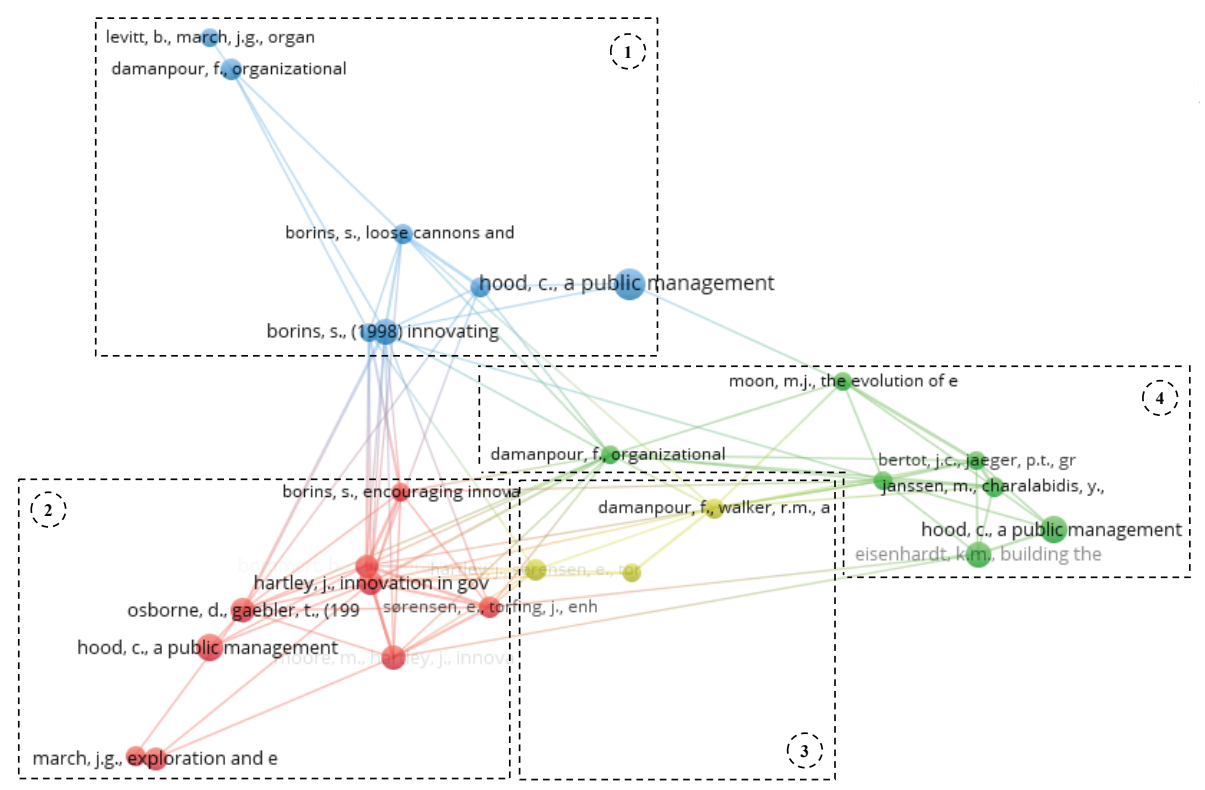

Fonte: elaborado pelos autores.

A referência "Hood, A. C. (1991) 'A public management for all seasons?' Public Administration, Vol. 69 No.1, pp. 3-19” aparece indevidamente nos clusters 1, 2 e 4, por estar declarada de formas diferentes entre as bibliografias usadas pelos documentos da base. Por outro lado, este dado reforça a relevância da obra para a pesquisa no campo. Analisaram-se os abstracts das obras em cada cluster para identificar linhas temáticas de pesquisa. O primeiro cluster dirige suas pesquisas para o papel de gestores públicos inovadores no incentivo à aprendizagem organizacional e à inovação. Com nove artigos, o cluster 2 foca em pesquisas sobre o papel da inovação colaborativa e do empreendedorismo para a promoção de inovações em governança e na burocracia do setor público. O terceiro cluster agrupa três obras e trata os processos de difusão dos diferentes ti- 
pos de inovação nas estruturas da gestão pública e seus efeitos. O último grupo, com oito obras, aborda o e-government, a participação cidadã e alguns aspectos culturais para sua adoção, como abertura e transparência.

\subsection{Rede de acoplamento bibliográfico}

A figura 6 mostra a rede para as 22 obras que compõem a primeira zona de trabalhos de maior impacto (conforme tabela 2). $\mathrm{O}$ tamanho de cada nó também representa o total de citações.

Figura 6 - Acoplamento bibliográfico

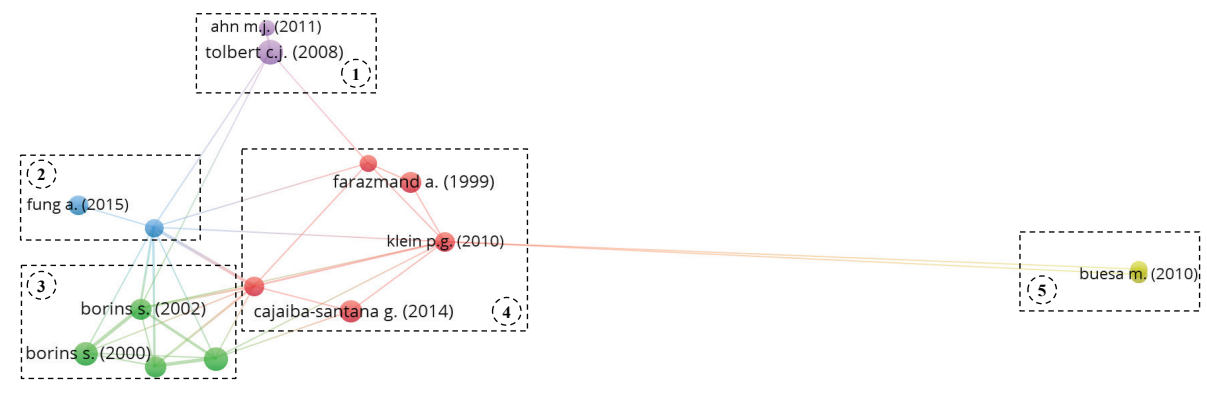

Fonte: elaborado pelos autores.

A relação de acoplamento bibliográfico entre duas obras é tão maior quanto maior o número de referências que elas compartilham, aproximando seus nós na rede. Dos 22 artigos em análise, sete não compartilhavam nenhuma referência bibliográfica com qualquer outro artigo, razão por que não aparecem na rede. Alguns dos documentos mais citados na base estão entre eles, como "Hicks D. (2012) 'Performance-based university research funding systems', Research Policy, Vol. 41 No. 2, pp. 251-261”, “Bossert T. (1998) 'Analyzing the decentralization of health systems in developing countries: Decision space, innovation and performance', Social Science and Medicine, Vol. 47 No. 10, pp 1513-1527”, e “Del Brio J.A e Junquera B. (2003) 'A review of the literature on environmental innovation management in SMEs: Implications for public policies', Technovation, Vol. 23 No. 12, pp 939-948. 
As principais temáticas identificadas nos clusters obtidos foram: e-government como inovação política para o controle político da burocracia (cluster 1), inovação colaborativa para a participação cidadã e governança pública (2), o papel dos líderes empreendedores na administração pública para a difusão de inovações e o desempenho organizacional (3), discussões teóricas sobre a influência da globalização, empreendedorismo e participação social na administração pública (4) e inovação na administração pública e sua relação com os demais atores no ecossistema de inovação (cluster 5). O evidente distanciamento do quinto grupo se deve ao fato de suas duas obras terem, cada uma, apenas uma referência em comum com uma das obras do cluster 4, sem a qual este grupo teria ficado isolado dos demais.

\subsection{Rede de co-ocorrências de palavras-chave}

A amostra contou com 1614 palavras-chave de autores, 45 das quais ocorrem pelo menos cinco vezes. A figura 7 exibe os relacionamentos de co-ocorrência entre elas. O tamanho dos nós indica a frequência de ocorrência e nós mais próximos refletem palavras que aparecem simultaneamente em um maior número de artigos.

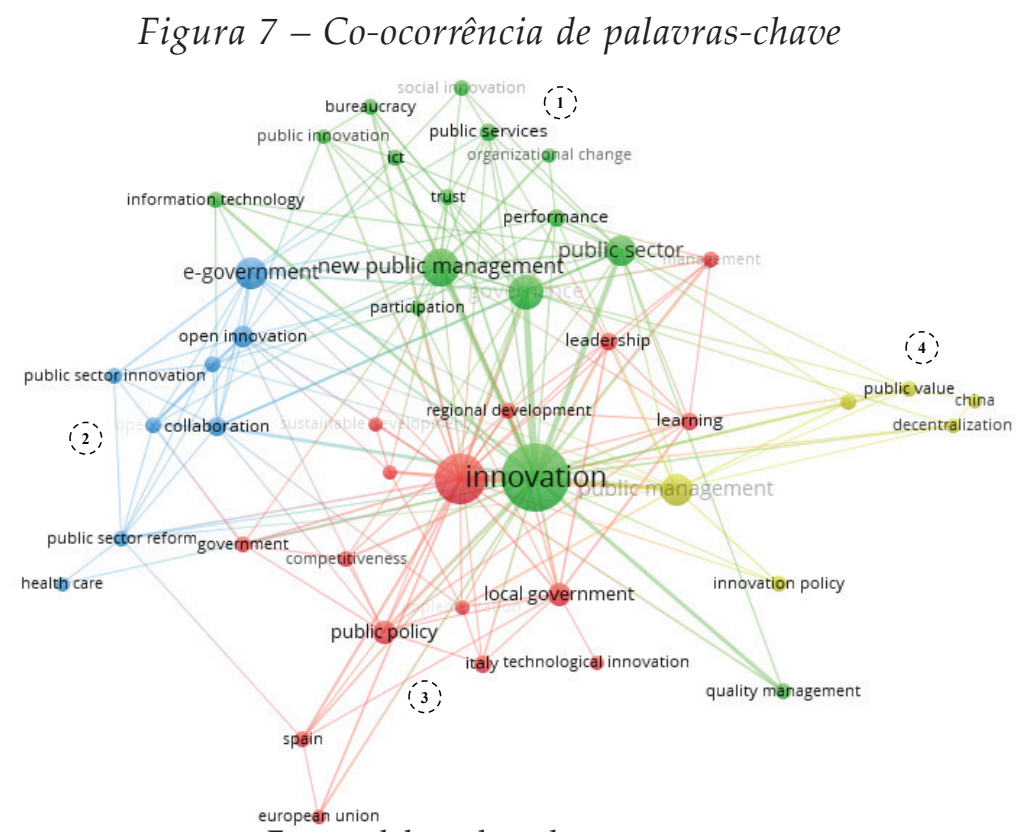

Fonte: elaborado pelos autores. 
As palavras-chave mais frequentes formaram uma rede com quatro clusters. O primeiro deles tem como principais palavras-chave "innovation", "new public management", "governance", "public sector" e "performance", reunindo pesquisas que tratam a perspectiva gerencialista da inovação no setor público, focada em desempenho, preceitos da Nova Administração Pública. O cluster 2 abrange pesquisas que lidam com questões mais técnicas voltadas a melhorias no setor público a partir de abordagens colaborativas, como a inovação aberta, enfatizando a também o e-government, termo com maior frequência no grupo. O terceiro cluster está centrado no termo "public administration" como palavra-chave mais frequente, seguido de "public policy", "local governerment", "leadership", "learning" e "regional development", além de reunir países ou blocos, como "Italy" e "European Union"; infere-se que seus estudos abordam inovações em políticas públicas empreendidas por governos locais e/ou o seu fomento por organismos multilaterais visando ao desenvolvimento. Por fim, o cluster 4 agrupa expressões como "public management", "innovation policy" e "sustainability", sugerindo estudos em práticas de inovação pública para a sustentabilidade.

\section{CONCLUSões}

O objetivo do presente estudo foi apresentar um mapeamento da produção científica internacional sobre inovação e gestão pública. O mérito do estudo reside na conjugação de duas abordagens: uma mais tradicional, em que se apresentam contagens de informações bibliográficas (evolução da produção, autores mais frequentes, obras de maior impacto, etc.), e uma abordagem sociométrica, menos usual, em que se discutem redes de coautoria, co-citação, acoplamento bibliográfico e co-ocorrência de palavras-chave.

Os resultados mostraram que a produção no campo apresentou uma consolidação nos últimos anos, estabilizando uma tendência de crescimento apresentada até o ano de 2015. As análises de autores mais frequentes e journals que mais publicam confirmaram os pressupostos das leis bibliométricas de Lotke e Bradford, respectivamente.

Com o suporte do software $\operatorname{VOSviewer}^{\odot}$, a análise de redes identificou os principais grupos de autores, as principais referências bibliográficas citadas, além de como eles se assemelham em 
termos de acoplamento bibliográfico. A rede de co-ocorrência de palavras-chave evidenciou o pressuposto da lei de Zipf, de que poucas palavras são muito frequentes em um texto. Todas estas análises permitiram identificar temáticas de pesquisas na área, como inovação e mensuração de performance na administração pública; abertura à inovação e e-government; inovação colaborativa e empreendedorismo; inovação, liderança e aprendizagem organizacional; relação entre gestão pública e outros atores no ecossistema de inovação; inovação colaborativa e participação cidadã; e inovação para a sustentabilidade.

O estudo possui algumas limitações que não puderam ser suplantadas: análises de rede aqui discutidas não consideraram a abordagem temporal (timeline-based approach), perspectiva que viabiliza uma maior compreensão do campo ao visualizar redes de citação direta entre os documentos em função do período no tempo em que foram publicados. Um processo mais lento e detalhado de categorizações a partir do conteúdo dos abstracts ou mesmo do texto completo de todos os documentos permitiria a triangulação com as temáticas identificadas através de análises de rede, ampliando a confiabilidade destes resultados. Tais áreas temáticas podem ser ponto de partida para futuras pesquisas.

\section{REFERÊNCIAS}

AGGER, A.; SØRENSEN, E. Managing collaborative innovation in public bureaucracies. Planning Theory, v. 17, n. 1, p. 53-73, 2018.

ALVARADO, R.U. A produtividade dos autores sobre a lei de Lotka. Ciência da Informação, v. 37, n. 2, p. 87-102, 2008.

ARUNDEL, A.; BLOCH, C.; FERGUSON, B. Advancing innovation in the public sector: Aligning innovation measurement with policy goals. Research Policy, v. 48, n.3, p. 789-798, 2019.

AXELSON, M.; NETZ, J.; SANDSTRÖM, C. Collective action problems in public sector innovation: A business model perspective. Creativity and Innovation Management, v.26, n. 4, p. 370-378, 2017.

BARTON CUNNINGHAM, J.; KEMPLING, J.S. Implementing change in public sector organizations. Management Decision, v. 47, n. 2, p. 330-344, 2009.

BOSSERT, T. Analyzing the decentralization of health systems in developing countries: Decision space, innovation and performance. Social Sciences \& Medicine, v.47, n. 10, p. 1513-1527, 1998. 
BRANDÃO, S.; BRUNO-FARIA, M. Inovação no setor público: Análise da produção científica em periódicos nacionais e internacionais da área de administração. Revista de Administração Pública, v. 47, n.1, p. 227-248, 2013.

BUFREM, L.; PRATES, Y. O saber científico registrado e as práticas de mensuração da informação. Ciência da Informação, v.34, n.2, p. 9-25, 2005.

COSTA SANTOS, M.J.V. Correspondência científica de Bertha Lutz: um estudo de aplicação da lei de Zipf e ponto de transição de Goffman em um arquivo pessoal. Ponto de Acesso, v.3, n. 3, p. 317-326, 2009.

DE VRIES, H.; TUMMERS, L.; BEKKERS, V. The diffusion and adoption of public sector innovations: A meta-synthesis of the literature. Perspectives on Public Management and Governance, v. 1, n. 3, p. 159-176, 2018.

DEMIRCIOGLU, M.A. The effects of empowerment practices on perceived barriers to innovation: Evidence from public organizations. International Journal of Public Administration, v.41, n. 15, p. 1302-1313, 2018.

FASSAUER, G.; SCHIRMER, F. Professionals as an implementation barrier to change in public organisations: Political and identity perspectives. International Journal of Leadership in Public Services, v. 4, n. 4, p. 11-23, 2008.

FERREIRA, F.; DE LIMA, S.; GOMES, A.; MELLO, G. Governança Corporativa: um estudo bibliométrico da produção científica entre 2010 a 2016. Organizações em contexto, v. 15, n. 29, p. 323-342, 2019.

HOOD, C. A public management for all seasons? Public Administration, v.69, n.1, p. 3-19, 1991.

KESSLER, M.M. Bibliographic coupling between scientific papers. Journal of the Association for Information Science and Technology, v. 14, n. 1, p. 10-25, 1963.

LIMA, D.H.; VARGAS, E.R. Estudos internacionais sobre inovação no setor público: como a teoria da inovação em serviços pode contribuir? Revista de Administração Pública, v. 46, n.2, p, 385-401, 2012.

MACHADO JUNIOR, C., SOUZA, M.T.S., PARISOTTO, I.S., \& PALMISANO, A. As leis da bibliometria em diferentes bases de dados científicos. Revista de Ciências da Administração, v. 18, n. 44, p. 111-123, 2016.

OSBORNE, S.P.; BROWN, L. Innovation, public policy and public services delivery in the UK: The word that would be king? Public Administration, v. 89, n.4, p. 1335-1350, 2011.

PEREIRA, C. S. T.; VIEIRA, A. M.; DAMIÃO, W. S. Dimensões da Inovação na Pós-Graduação: Papéis e Significados. Revista Organizações em Contexto, v. 14, n. 27, p. 211-234, 2018.

ROCHA, A. F. R.; SANTOS, I. C. D.; VIEIRA, A. M. Semi-open Innovation: an Approach to the Innovation Typology. Future Studies Research Journal: Trends and Strategies, v. 10, n. 1, p. 55-81, 2018.

RUEDIGER, M.A. The rise and fall of Brazil's public security program: PRONASCI. Police Practice and Research, v. 14, n. 4, p. 280-294, 2013. 
SERDYUKOV, P. Innovation in education: What works, what doesn't, and what to do about it? Journal of Research in Innovative Teaching \& Learning, v. 10, n.1, p. 4-33, 2017.

SMALL, H. Co-citation in the scientific literature: a new measure of the relationship between two documents. Journal of the American Society for Information Science, v. 24, n.4, p. 265-269, 1973.

SMITH, G.; SOCHOR, J.; KARLSSON, I.C.M. Public-private innovation: Barriers in the case of mobility as a service in West Sweden. Public Management Review, v. 21, n.1, p. 116-137, 2018.

VAN ECK, N.; WALTMAN, L. Visualizing bibliometric networks, in Ding, Y., Rousseau, R. e Wolfram D. (Orgs.), Measuring scholarly impact: methods and practice, Springer, New York, NY, p. 285-320, 2014.

VANTI, N.A.P. Da bibliometria à webometria: uma exploração conceitual dos mecanismos utilizados para medir o registro da informação e a difusão do conhecimento. Ciência da Informação, v. 31, n. 2, p. 152-162, 2002.

VOLACU, A. Justice, efficiency, and the new public management. Australian Journal of Public Administration, v. 77, n. 3, p. 404-414, 2017.

Recebido em: 5-6-2019

Aprovado em: 17-11-2020

Avaliado pelo sistema double blind review.

Disponível em http://mjs.metodista.br/index.php/roc 